\title{
The role of attention and intention in dreams
}

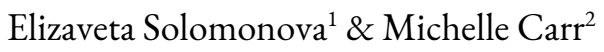

${ }^{1}$ Neurophilosophy Lab, Division of Social and Transcultural Psychiatry, McGill University

${ }^{2}$ Sleep and Neurophysiology Research Laboratory, Department of Psychiatry, University of Rochester

*This is a draft version, please cite the published version when available.

To appear in: Access and Mediation: A New Approach to Attention. Wehlre, M., D’Angelo, D. \& Solomonova, E., eds (forthcoming, 2022) De Gruyter

https://www.degruyter.com/document/isbn/9783110647242/html

\section{Introduction}

I was walking along the seafront. It was snowing lightly. The sea was frozen over. I decided to skate on the frozen sea and went down to the beach. I was suddenly wearing ice skates. I started skating out over the sea in a straight line away from the coast line. I reached an island and took a rest. It was very warm but the sea was still frozen. I laid down on the white sand in the sun. (Carr et al, 2020)

Traditionally, dreams have been seen as experiences that one cannot control, as something that happens to the dreamer (at times through involvement of supernatural powers), without the dreamer's permission, volition or agency. This view was famously challenged in the advent of psychoanalysis: in his Interpretation of Dreams, Freud proposed that while we may not be consciously in control of our dreams, our unconscious mind is actively constructing dream content, and that dream content is symptomatic of our repressed, accumulated neuroses (Freud, 1900/2010). This shift in perspective signaled that dreams may in fact be subject to individual experience and to one's mental state (which is something that is possible to change, or even to control), therefore bringing, at least partially, responsibility for dreams to the dreamers themselves. Emerging neuroscience of dreams, in 1970s, however, adopted a more conservative behaviorist position, and for a relatively long time the dominant view has been one of neuro-reductionism, where dreams were seen as random hallucinatory products of the activity of the sleeping brain (Hobson \& McCarley, 1977). In recent years, a more 
nuanced picture of dreams is gradually emerging. Research from psychology, philosophy and anthropology converges on the idea that dreams may be individual or even collective practices rather than uncontrolled brain events. Developmentally and temporally, dreaming can be recognized as cognitive achievement (alongside other cognitive abilities, such as memory, perception, attention, etc.) (Foulkes, 2014). And dream qualities, including what is possible in the dream state, how rich the dream experience is, and how well the dream will be remembered, may change as a result of attentional practices during waking hours. Research on dream incubation, dream sharing and lucid dreams shows that the dreamer is an active participant and co-creator of their dream life, and that the dreamer's agency, awareness and degrees of control are all dynamic, continuous and potentially trainable skills. Further, in line with work on $4 \mathrm{E}$ cognition (Menary, 2010) and following evidence from sensory incorporation studies (Nielsen, 1993, 2017; Sauvageau, Nielsen, \& Montplaisir, 1998), it has been proposed that dreams are not simply experiences of virtual reality confined in the sleeping brain, but rather can be conceptualized as processes of embodied imagination (Thompson, 2014; Solomonova \& Sha, 2016; Solomonova, 2017), rooted in lived sensorimotor experience and responsive to sensory information from the outside world.

In this chapter, we review the different ways that attention works in relation to dreams and how it may function in dreams, and apply the framework of attention, proposed in this volume - as a means of accessing and mediating interactions with the world - to the dreaming world. We first review prior work on the role of attention as 1) access to dreams, e.g., how practices of recording and sharing dreams act as enabling factors for improving dream recall and enhancing richness of dream experience; and as 2) a mediator of dreams, e.g., how incubation, imagery rehearsal, and ultimately lucidity can be cultivated as cognitive skills enabling agency in the dream experience. We propose that attention functions as a constitutive factor in dream experience and that it is a trainable, developmental cognitive skill. We argue that dreams are not simply experiences that happen to the dreamer, rather, through employing attentional techniques in various ways, the dreamer may cultivate different degrees of agency in the dream.

\section{Section 1 - Attention: Access to the dream state}

In this section we will discuss how attention facilitates and improves access to the dream state. First, we will show how attention to dreams (or to memory of dreams) during waking state shapes our capacity to have vivid dreams and our ability to report and describe the dream experience. Second, we will review work on attention in dreams during sleep, and will suggest that attentional processes during sleep reveal quasi-perceptual creative processes of dream imagination.

\subsection{Paying attention to dreams during waking life}

In researching dreams one of the fundamental methodological difficulties lies in the fact that we do not have direct access to the dream state; instead, we rely on the dreamer's memory for the dream experience and on the dreamer's capacity to report on their experience. A number of things must happen before dream researchers have access to a dream narrative, and all of them rely on various forms of attention and memory. First, the dreamer must be able to remember the dream as it happens during sleep. To do so, the dreamer's attentional skills during sleep must be sufficiently active in order to be able to be engaged in the dream scenario and to commit the dream experience to memory. In the majority of dreams these attentional skills do not require 
recognizing that the dream is a dream. The dreamer then largely makes use of habitual patterns of attending to the world, applying them to the dream experience. The dream state, however, has its own rules, and attention in dreams must adapt to the altered neurobiological state of sleep. We will discuss some of the unique phenomenology of the dream experience further in this chapter. Second, the dreamer must change neurobiological state and transition from sleep into wakefulness. During this process there is risk that the dream may be forgotten or distorted due to interference from other stimuli. Third, the dreamer must be able to narrate the dream bearing in mind the dream's temporal structure, emotional tone, visual, auditory, somatosensory and other aspects. This requires sustained awake attention to the fragile and quickly dissipating memory of the dream. Little work to date has focused on attention in context of dreams, likely due to the limitation that it is nearly impossible (unless one is in a lucid dream, which is discussed in section 2 of the present chapter) to study attention experimentally in the dreaming subject.

In relation to neurocognitive bases of dream recall, some evidence points to the notion that remembering dreams may be a function of an individual attentional profile. For instance, Conduit and colleagues (Conduit, Crewther, \& Coleman, 2000), proposed that dream recall is not a function simply of having had or not having had an experience during sleep, but is dependent on activation of attentional circuits during dreams, which, then, enables the dream to be attended to, encoded in memory and then remembered upon awakening. Similarly, work in Ruby's group revealed that individuals who claim to have higher frequency of dream recall, exhibit specific traits in brain activity and brain architecture in areas associated with attentional, motivational and memory processes (higher white matter density and cerebral blood flow in medial prefrontal cortex, higher cerebral blood flow in temporo-parietal junction) (Eichenlaub, Nicolas, et al., 2014; Vallat, Eichenlaub, Nicolas, \& Ruby, 2018), as well as larger attentional-orienting responses (evoked potentials, brain signatures signaling that an experimental stimulus was attended to) during wake (Eichenlaub, Bertrand, Morlet, \& Ruby, 2014).

Empirical research on dream recall, as well as a wealth of popular wisdom, shows that attending to dreams (whether by keeping a dream journal or by cultivating positive attitudes towards dreams) improves dream recall (Aspy, 2016; Beaulieu-Prevost \& Zadra, 2004, 2007; Schredl, 2002). In other words, paying attention to dreams (or to memory of dreams) while awake increases one's capacity to remember future dreams, possibly improving ability to pay attention (and to engage memory-encoding processes) while in a dream. The most common advice given to those who wish to remember more dreams is to get into the habit of recording dreams first thing in the morning. In addition to this, dreamers are also often encouraged to recall and mentally rehearse any previous dream prior to going to bed, and to set intention, to remind themselves, that they must remember their dreams upon awakening. These practices are very efficient, but raise two important questions: by changing the way one attends to their dreams, does one reveal a dream life that was already happening but was forgotten? Or does one effectively change dreams and create a richer and more memorable dream life by virtue of intending to attend to the dream experience, and thus learning to more effectively deploy attentional and memory-building abilities while in the dream state?

An important methodological difficulty in dream research is related to the fact that dreams are not restricted to the stage of the rapid eye movement (REM) sleep, as previously thought, and may happen at any moment and in any sleep stage. This led some scholars to hypothesize that perhaps we always dream, and only when we are able to pay attention to the dream and therefore to engage memory encoding mechanisms, that we remember a dream. On the other end of the spectrum, Dennett's “cassette theory" of dreams (Dennett, 1976) 
suggests that dreams are not happening in real time at all and are constructed retroactively by the dreamer upon awakening. This latter framework presupposes that during sleep there is nothing to pay attention to. One example of the question of whether or not dreams are happening somehow undetected by the reflective consciousness unless one learns to pay attention to them, comes from research on what is sometimes termed "white dreams". White dreams are dream reports that contain only a feeling of having had a dream, but no memory of sensory experience or narrative. Traditionally, white dreams have been considered to be dreams that are forgotten, i.e., the assumption is that if one has a memory of "something", it must have been a full dream, which is now forgotten due to interference of other stimuli upon awakening or insufficient attention to the dream. This reflects a bias in the literature and popular view of the dreams which represents "real" dreams as fully immersive spatio-temporal and audio-visual experiences. A recent paper (Fazekas, Nemeth, \& Overgaard, 2019) challenges this assumption and offers an alternative explanation: white dreams are full experiences of low perceptual content (less vivid, less clear and less stable than most waking and dream experiences) which are then remembered as such upon awakening. Similarly, while "deep” sleep (NREM stage 3 sleep) has traditionally been seen as "dreamless" sleep, more careful empirical examination of cognitive phenomena associated with this stage of sleep has revealed a variety of diminished forms of dreaming (Windt, Nielsen, \& Thompson, 2016). These include, on the one hand, dramatic sleepwalking or night terror experiences, and, on the other hand, minimal states of awareness and potentially contentless experiences (different from unconsciousness) of "having slept", what Windt referred to as "pure temporality" (Windt, 2015b). These examples highlight a tension between the opposing possibilities that either a) we dream all the time but sometimes (as in deep sleep) our attentional capacities are not "on" enough therefore we do not remember our dreams; or b) dream reports reflect exactly what the experience was, and when we do not remember, in fact, we do not dream.

Another context in which attention to dream reports matters is in practices of dream interpretation and dream sharing. In this case, attending to memory of dreams and constructing and rehearsing a dream narrative serves not only as a future dream-enhancing cognitive tool, but also plays an important social role. By bringing dreams into the shared space of narrative and interpretation, it was suggested (Blagrove et al., 2019) that attending to and sharing dreams with others has a social bonding function and potentially increases empathic response towards the dreamer. Similarly, attending to dreams in context of psychotherapy has been shown to increase connection and trust between therapists and patients (Hill \& Knox, 2010; Hill et al., 2014; Pesant \& Zadra, 2004).

Lastly, there is a growing body of evidence that attention training during wakefulness may change attentional processes in dreams, in particular with regards to increasing qualities of lucid dreams. For example, Baird and colleagues (Baird, Riedner, Boly, Davidson, \& Tononi, 2019) found that long-term meditators (but not novices) had higher frequency of lucid dreams. Similarly, dispositional mindfulness (a trait predisposing an individual to perceive the world with increased awareness) was associated with an increase in lucid dreaming (Stumbrys, Erlacher, \& Malinowski, 2015). Developing lucidity during sleep is also an integral part of the Tibetan Buddhist training. The practice of dream yoga (Holecek, 2016; Norbu, 2002; Wallace \& Hodel, 2012) is aimed at cultivating awareness throughout the night. Specifically, the practitioners are learning to use attentional skills acquired during waking meditation practice while dreaming in order to gain insight into the nature of the mind and to advance on contemplative path. 


\subsection{Paying attention while dreaming}

Much work has been done in psychology and phenomenology of perception with regards to how an individual may learn to attend to aspects of the world. Elements of the environment that may attract attention may be salient, surprising, motivating, engaging. The enactive approach in cognitive science and philosophy (Gallagher, Hutto, Slaby, \& Cole, 2013; Noe, 2006; Stewart, Gapenne, \& Di Paolo, 2010; Varela, Thompson, \& Rosch, 1992) teaches us that the act of perceiving the world is not a passive input-output information processing activity, and that there seems to be a clear relationship between one's experience and the way that one is oriented towards or, in Merleau-Ponty's terms, geared towards the world (Merleau-Ponty, 1945). In other words, we see what we have learned to see, and we engage with the world in the way in which we have habituated to meet it. But what about dreams? How does a world, brought forth by the dreamer, anchor and mobilize attentional capacities? How does the dream or the dreamer determine what to pay attention to and which elements of the experience will be remembered? Some debates, both in philosophy and in cognitive science of dreams, have focused on the question whether dreams are more like perception or more like imagination (for a review of the literature see (Windt, 2015a).

In waking visual perception, our eyes are constantly making rapid jerky movements, known as saccades (Hutton, 2008). These movements correspond to what we are attending and orienting to in visually rich environments. During the Rapid Eye Movement (REM) stage of sleep, associated with the highest rates of immersive, vivid and complex dream experiences (Nielsen, 2000), even though the body is paralyzed, the eyes are rapidly moving (Aserinsky, 1965), resembling the activity during waking perceptual saccades. One idea aiming to explain the reason for such movements is known as the "scanning hypothesis" (Herman, Barker, \& Roffwarg, 1983), which suggests that these eye movements represent quasi-perceptual activity during dreams mapping on to what the dreamer is looking at. Indeed, some research (Arnulf, 2011) suggests that eye movements during sleep may, in fact, correspond directly to reports of the direction where the dreamer was likely looking during the dream. In other words, eye movements represent an idiomorphic relationship between attentional acts during wake and during dreaming, suggesting that both the experiential aspects and the dreamer's orienting and attending behavior during dreaming are very similar to that during waking. Recent work by LaBerge and colleagues (LaBerge, Baird, \& Zimbardo, 2018) aimed at clarifying some aspects of the debate around whether dreams were more like perception or like imagination. Experienced lucid dreamers (dreamers who learned to become aware of the dream state while in a dream) were asked to trace a shape with their finger and to follow the finger with their eyes. In waking cognition, attending to an external stimulus is reflected in smooth eye movements, while imagining that same stimulus produces jerky eye movements. Lucid dreamers, when following a dreamt stimulus with their eyes, showed a smooth pattern of eye movements, thus suggesting that the dreamt environment, even if endogenously brought forth, still has experiential qualities and attentional response similar to that of the external world.

While there are clearly some similarities in the way in which we attend to the world around us while awake and while dreaming, there are also some important nuances in how attention and perception function during dreaming which are not quite so similar to waking life. In fact, in waking life, whether we are paying attention or not, the world makes demands on our perceptual system and even stimuli that are not consciously attended to are often parts of a more general sense of awareness of the environment. In dreams, however, it appears as if the entire dream reality directly depends on attention. In Thompson's account of dreams as 
processes of enactive imagination, he writes: "If I don't notice trees in my flying dream, is that because I fail to notice their presence or because I'm not generating any sensory imagery of trees? It strikes me as more phenomenologically accurate to say that the reason I don't notice them is that I'm not visually imagining them at the moment" (Thompson, 2014, p.182).

Further, paying attention to the dream world during a non-lucid dream (a dream in which the dreamer is not aware of the fact that they are dreaming) often does not result in the realization that the dream content is implausible/bizarre (as compared to waking life). Indeed, relatively high prevalence of plot discontinuities, instability of characters and objects, scene changes, and narrative jumps in dreams (Mamelak \& Hobson, 1989; Rittenhouse, Stickgold, \& Hobson, 1994) all attest to the idea that it is quite difficult to sustain attention on a stimulus or a task during a dream. This fact has often been used to suggest that "failure" to realize that a dream is bizarre represents a form of cognitive deficiency, akin to psychosis (Dresler et al., 2014; Hobson \& Voss, 2011). An alternative view would imply that in dreams we are perhaps pre-reflexively aware (even if not explicitly lucid or rational) of the fact that we are dreaming. Thus, our dreamworlds are familiar to us, and are organized differently with different kinds of affordances (e.g., we can fly or spend time with deceased loved ones without being delusional). Dreaming is one of the earliest forms of cognitive life, likely starting at the same time as waking cognition. Some evidence for this notion comes the developmental trajectory of dreaming-rich REM sleep, forms of which develop in already in utero and are abundant in newborns (Roffwarg, Muzio, \& Dement, 1966). It is unclear, therefore, why dream life would have to be subject to the same criteria for rationality, realness or plausibility as waking life. In our waking life, too, much time is quite productively spent on "implausible" or "bizarre" pursuits, ranging from private experiences of daydreaming or imagining to public and collective practices of art, filmmaking and literature. Dreams, we argue, are better understood as imaginary lives and performative processes with their own fields of affordances and with their own personalized experiential vocabulary for what is possible, it does not and nor does it need to map directly onto waking life.

Another way in which the dreamer's attention may be hijacked or reoriented in the dream, is through incorporation of outside stimuli into dreams. Studies of sensory incorporation into dreams show that the dreaming mind reacts to the physical world in a variety of ways (Nielsen, 1993, 2017; Sauvageau et al., 1998; Schredl et al., 2009; Solomonova \& Carr, 2019). Interestingly, stimuli only rarely appear directly in the dream, rather, this new intruding information is often transformed by the dream into something else, which may more or less fit within the ongoing dream scenario. Furthermore, stimuli may change their modality upon entering the dream world: somatosensory stimulation may produce a change in visual or auditory experience. In the dream examples below, the dreamers were undergoing a somatosensory stimulation protocol, which consisted of inflating a blood pressure cuff on the participant's ankle during sleep, followed by a dream interview (Solomonova, 2017).

Liza was there to wake me up. She turned on the lights and asked me about my dreams. I was answering her. I could feel the pressure pump on my leg. She asked me what does it feel like, I said it feels like a bug. She said 'Doesn't it feel like someone pulling on your leg?'

... I was in my parents' car ... and a large marine animal approached me as if he knew me. It wanted to play with my purse and it bit me! I didn't want (it to continue) and I was saying Ob No!* 
These excerpts show two different dreamt responses to the intrusion of the somatosensory stimulus (pressure on an ankle). In the first one, the dreamer immediately associates the sensation of the pressure cuff with the ongoing protocol in the lab (they are expecting this to happen), and then dreams of the experimenter, imagines giving a dream report and describes the phenomenology of feeling the pressure cuff inflate on their leg, in a somewhat humorous manner. In the second example, the pressure cuff intensified the ongoing dream. It produced a dramatic scene shift (from a parents' car to encountering a large animal), a confrontation with a threatening animal, and a physical sensation of being bitten. In both cases, the external stimulation was processed by the brain and transformed by the dreamer into something that was part of the ongoing dream experience. Thus, the dreamer's attention was shifted from what was going on in the dream prior to the stimulation and was now focused on integrating this new and demanding stimulus into the dream. This suggests that the stimuli we attend to in the dream are processed in a multisensory (Nielsen, 2017) and perhaps even synesthetic manner, and are in line with contemporary theories of dream formation that suggest that dreams function in an associative (Hartmann, 1996; Horton \& Malinowski, 2015) manner, bringing together disparate elements from memory. Moreover, examples from somatosensory dream incorporation highlight the role of the body in dream formation, lending further support to the idea that dreams are not happening exclusively "in the head" but are indeed, as the enactive view of the mind would suggest, forms of embodied imagination (Thompson, 2014).

In this section we discussed the ways in which attention to dreams during wake and attention in dreams during sleep facilitates access to the dream world. Attentional practices in wake and dreams also have potential for insight in modulating the dreamer's agency. In the following section we will discuss how attention and intention function as mediators of dream content.

\section{Section 2 - Mediation: intention, and agency in the dream}

\subsection{Agency over dreams}

It has been proposed that attention and intention play a role in skilled agency (Wu, 2016). However, fundamental questions of agency over dreams remain largely unanswered. Does the dreamer direct the dream or does the dream direct the dreamer? On the one hand, the dreamer is the source of the dream, i.e., everything that unfolds in the dream is formed by the dreamer's mind. On the other hand, the dreamer is often left at the mercy of the dream with little or no control over the dream scenario. In Section 1 we discussed how attention in dreams functions as a way to bring forth the dream environment. In this section we will discuss the different aspects of how a dreamer's self may be instrumental in the oneiric narrative through practices of intention and skilled attention to and in dreams.

Traditionally, dreaming is thought of as an experience that happens to the dreamer, and over which the dreamer has very little agency or control. In support of this view, to some extent, are the cognitive and neurophysiological evidence, suggesting that during dreaming one has relatively less cognitive control than during waking, due to relative inhibition of frontal cortical activation during REM sleep (Cicogna \& Bosinelli, 2001; Rechtschaffen, 1997). In line with this, dream reports demonstrate relatively less cognitive agency compared to waking or even daydream reports. In this context, cognitive agency refers to whether one's thoughts seem to be controlled or directed by the dreamer, also termed "reflective" or "metacognitive" thoughts. 
To illustrate, below is an example of a daydream report, which while imaginative still demonstrates the intact cognitive agency more typical of waking thought.

I was thinking about being calm and the image of a small forest came to mind. I thought about walking through that forest with my closest friends and what we would talk about and how safe and happy I feel with them. Spending time with them motivates me to work on my art projects. I then started thinking about all the projects I have going and what I'm going to work on later today. (Carr \& Nielsen, 2015)

In general, cognitive agency is lower in dream reports, whereas immersive and embodied imagery is clearly higher in dreams than in either daydream or waking reports. Speth and Speth (2018) suggest that in dreaming, "to think is to do," meaning rather than reflecting on or directing thoughts and actions, the dreamer simply reacts to and interacts with the dream imagery in a relatively impulsive and non-reflexive way.

Many dream researchers have claimed that such a temporary suspension of reflective thought (akin to Coleridge's "suspension of disbelief”), e.g., a general inability to recognize that one is in a dream, or acceptance of dream scenario despite its implausibility or bizarreness, allows us to become fully immersed in dream imagery, and may enable functional outcomes associated with dreaming, including emotion regulation (Cartwright, 2011), memory consolidation/integration (Horton \& Malinowski, 2015; Nielsen \& Stenstrom, 2005; Wamsley \& Stickgold, 2010), insight (Edwards, Ruby, Malinowski, Bennett, \& Blagrove, 2013), among others. For instance, Freud (1900) famously suggested that the fact that dreams are bizarre and therefore "unreal" provides the dreamer with a "safe space" to enact their repressed anxieties and traumas, thus allowing for a cathartic emotional release, while being non-threatening to the ego, since the manifest content of the dream has undergone many transformations and distortions. These distortions, according to the psychoanalytic view, hide the real, latent, source of the dream.

From another angle, dreaming has been related to a form of involuntary autobiographical memory, which likewise occurs during daydreaming and mind-wandering (Rasmussen \& Berntsen, 2011). Involuntary autobiographical memory is triggered by associative and context-dependent retrieval and requires little executive control (Singer, 1966; Smallwood \& Schooler, 2006). Involuntary autobiographical recall tends to occur during periods of unfocused attention, such as daydreaming and dreaming, compared to voluntary recall, which is more frequent during goal-directed cognition. Support for a link between involuntary autobiographical memory and dreaming is shown first by evidence that more than $80 \%$ of dreams contain autobiographical memory features (Malinowski \& Horton, 2014), and second by a phenomena termed the 'dream rebound', that suppressing thoughts before sleep prompts their involuntary incorporation in dream content (Wegner, Wenzlaff, \& Kozak, 2004). These findings support that there are some important 'involuntary' processes underlying dream generation. Nevertheless, there is also evidence that intentional and voluntary pressures influence dream content, and that levels of agency within dreams vary along a continuum.

Co-creative dream theory acknowledges, on the one hand, contributions of a somewhat autonomous or involuntary process of dream imagery generation, and, on the other hand, a role for dreamer agency in dream content creation (Sparrow, 2014). Specifically, the theory suggests that even in non-lucid dreams, where the dreamer does not appear to have explicit awareness of or control over the dream, the dreamer is actively cocreating the dream by making specific choices or taking specific actions within the dream. Co-creative theory regards the dream as an interactive process between the dreamer as an agent and the unfolding dream narrative, 
thus the dreamer is an active and determinant participant in the process of dreaming. To some extent, this theory is supported by other research that acknowledge the presence of a range of levels of awareness and metacognition in ordinary, nonlucid dreams, in which the dreamer can reflect on and determine how to engage with the dreamworld, contrary to claims that reflective awareness is completely absent in dreaming (e.g., the Metacognitive, Affective, Cognitive Experiences)(Kahan \& LaBerge, 2011; Kahan \& Sullivan, 2012; Kozmová \& Wolman, 2006). Such approaches acknowledge the varied levels of directed attention and controlled behaviors a dreamer can have, guiding the ongoing dream narrative through choices, and actions. The dreamer is capable of directing the dream's development, in a dynamic and reciprocal process of dream creation (Sparrow \& Thurston, 2010).

Another recent approach suggests that simply 'feeling', the private mental experience of emotions (Damasio et al., 2000), informs attention and behavior in the world (Hoeksma, Oosterlaan, \& Schipper, 2004). For example, if undesired anger arises, specific behaviors such as gaze aversion (looking away from the object of unpleasant emotions) or physically changing the situation (i.e., leaving a room) are common responses enacted to regulate emotion, particularly in social interactions (Nummenmaa \& Calder, 2009). If the entire dream experience depends on where attention is directed, the affective tone of the dream, then, mediates attentional quality. In dreams, gaze aversion or gaze shifts from what one was initially paying attention to often occur as novel elements appear in the dream, or as narrative jumps that commonly occur as changes in dream scenes or situations. These shifts and the continual injection of novel elements into the dream may to some extent allow the dreamer to witness and interact with emotionally laden imagery without becoming overwhelmed. Several theorists (Nielsen \& Levin, 2007; Stickgold, 2005; Stickgold \& Walker, 2013) support this as a function of dreaming, to replay emotional memory in combination with novel associative elements, as a means of integrating salient experiences into broader autobiographical memory. However, this process is often interrupted by intense emotion, as evident in the case of nightmares (Nielsen \& Levin, 2007; Nielsen \& Carr, 2017). The nightmare seems to demonstrate a process of maladaptive emotion regulation strategies, particularly attentional narrowing, i.e., maintaining attention on an aversive stimulus. In such a case, the focus on a threat in a nightmare takes on a form of perseverative loop, where it appears that difficulty in voluntarily redirecting attention somewhere else leads to an amplified persistence of threatening or disturbing imagery. In this situation, the dreamer's attention and therefore their entire experience is "locked" onto the dysphoric scenario.

I was walking along the beach and it was raining lightly. The sun was setting over the ocean and I thought this was weird, as it was setting in the south and as I for some reason felt it was the middle of the day still. I watched the sunset until the sun was gone completely. Suddenly it was very dark, there were no visible stars, I couldn't see the moon, and there weren't any street lights. I didn't know which direction to walk in to avoid ending up in the sea. I started walking but slipped over and ended up in the water. I tried to swim to the surface but I couldn't figure out which way to go so just went what I thought was upwards. My lungs were burning and I couldn't reach the surface, everything was still dark and I felt dizzy and felt my eyes close. I'm fairly certain I died. (Carr et al, 2020)

While negative thoughts and attention can result in increasingly negative, narrowed and repetitive content, the complementary position may be true for intense positive emotion. That is, it broadens mindsets, increases openness to others, encourages exploration, and results in vivid and elaborate dreams (Carr \& Nielsen, 2017). In contrast to "locked" qualities of dream attention in nightmares and bad dreams, archetypal, or "Big", 
dreams tend to describe euphoric, integrative, altered state experience that demonstrate the expanding influence of positive emotion within a dream (Spadafora \& Hunt, 1990).

My family and I went to this amazing sight seeing place. It was on the top of a massive mountain so when you looked out it looked like it was the edge of the earth. You could see the stars in the darkness above the clouds and the clouds were pink and orange and they were falling off the mountain like a water fall. It was so stunning. We then went on a belicopter ride and it took off over the edge of the mountain and then there was nothing beneath you. I got the same feeling you get on a scary rollercoaster and like my stomach was in my mouth. I felt happy when I woke up. (Carr er al, 2020)

In these examples, the dreamer's emotional appraisals and attentional focus seem to direct the developing dream narrative, leading to the manifestation of anxiety in the case of the first dreamer who is fearful of falling into the ocean and then being unable to surface, yet leading to an aesthetically vivid and positive experience in the case of the latter dreamer who is filled with awe. Thus, the quality of attention, thoughts and habitual reactions of the dreamer partly shape the dream narrative. Through changing patterns of thinking or reacting in waking life, e.g., cultivating positive emotion, practicing adaptive emotion regulation strategies, etc., we can influence how and where attention is applied during dreaming. More intentional practices of attention can also be used to direct and exert varying degrees of control over dream content.

\subsection{Intentional practices using attention to change dreams}

Dream incubation is the practice of setting an intention prior to sleep in order to influence dream content. While dream incubation has been practiced for millennia as a means of guiding dreams to provide insight around social, cultural or personal problems, experimental research also shows that reflecting on current concerns prior to sleep can effectively incubate relevant dream content and even provide novel solutions. Barrett (1993) asked college students to incubate a specific, personally relevant problem of their choosing. After simply thinking about the problem for 15 minutes before sleep, participants rated $49 \%$ of their dreams as related to the problem and $34 \%$ of them as containing a solution. Saredi and colleagues (1997) also report that for a small sample of participants in a sleep laboratory study, reflecting on a question related to a significant current concern prior to sleep increased the frequency of dream references to the concern.

Imagery rehearsal (a form of dream incubation) and other types of dreamwork (working with dreams while awake through different intentional practice such as visualization or contemplation of remembered dreams) can introduce elements of change into recurring dream patterns, with some element of agency in the dreamer but not full lucidity. Imagery rehearsal therapy (IRT) is a promising treatment for frequent, distressing, or recurring nightmares (Aurora et al., 2010). This treatment focuses on rewriting a nightmare scenario during wake, typically writing down an alternate, more positive ending to the nightmare, and then mentally rehearsing the modified version of the dream several times prior to sleep. The principle of IRT is the idea that individual dream patterns, including nightmares, are examples of learned cognitive behavior, which have the potential of being unlearned and changed. Thus, working with and visualizing alternative nightmare narratives during wake helps "decondition" the dreamer and allows for new possibilities for the otherwise locked negative dream scenario. This simple technique is effective at treating nightmares and reduces both the frequency of nightmare experience and mental distress associated with nightmares (Krakow \& Zadra, 2006). 


\section{Dream Report:}

I'm put in a [prison] cell. [...] The cell has big windows that look down into a kitchen area that's dark and quiet [...] I'm looking through the window and I can see two kitchen drawers opening by themselves, as if it's by a ghost [...] It's a feeling of dread, I'm really frightened, my bairs go up and I'm cold...I just start crying, and I panic then.

\section{Re-scripting the Dream:}

I think if there were other people in the room, I'd feel better. [...] I feel like I could bold their bands, and I wouldn't be on my own. Just feels safer with other people. [...] the drawers are going back on their own now, we're not pushing them, we're just kind of standing there-like holding hands... and the drawers are going back.... and it feels a bit like when a room stretches, goes longer [...] it feels like, that's quite powerful...it's like winning.... and I can see the floor, the tiles, dark tile, light tile...checkered...I can see more of that...[...] As it's going away....the fear is going away.... and it's replaced with like "wow...this is working" [...]I feel calmer... and like lighter in the sense of weight, but also light-as in I would be a different shade of, it's gone from grey to shades of blue...I'm like a pale blue now. (Carr et al, 2020)

The re-scripting exercise allows the dreamer to imagine a more positive resolution to the dream even in the waking state. Thus, intentional practices harnessing attention in waking life can influence dream imagery and elevate the dreamer's agency in shaping the narrative. Specifically, it appears that the ability to recall, bring to attention and manipulate imagery in space of one's own imagination while awake, increases one's capacity for responding in a different way when facing spontaneous and uncontrolled imagery during dreams. Beyond this, it is possible to experience wake-like agency in a dream, through the practice of lucid dreaming, that is, becoming aware of the fact that you are dreaming while still asleep.

\subsubsection{Forms of attention and agency in lucid dreams}

Lucid dreams are unique in that the dreamer does have awareness of the fact that they are dreaming, enabling the dreamer to exert volition over the dreaming self and at times over the dreamworld. Lucid dreamers can choose how to attend to and respond to dream imagery moment-to-moment. Lucid dreaming offers a means of directing dreams from within the sleep state. Although there are individual differences in spontaneous lucid dreaming frequency, there are techniques individuals can use to induce lucid dreams and increase lucid dreaming frequency (La Berge, 1980). A few attentional techniques can be practiced during the day and just prior to sleep to increase dream lucidity. One technique, 'reality checking,' involves asking yourself repeatedly throughout the day whether you are currently dreaming, and this habit can then spill over into sleep and lead to the realization that one is dreaming. Another method involves setting the strong intention to become lucid by repeating an affirmation in mind prior to sleep such as, "The next time I am dreaming I will remember that I'm dreaming." This can be combined with visualizing a recent dream and imagining becoming lucid within the dream. These techniques are thus similar to dream incubation and imagery rehearsal methods, in that they utilize waking attention to influence dream content, but the goal is to then become lucid and able to further control the dream while asleep.

As discussed above, comparisons of dreaming with waking attention have typically focused on claims that the dreaming mind lacks the self-reflective awareness and metacognition that are assumed in wake. However, even in waking we often wander around in a state of semi-attention, simply perceiving and reacting to experience without affording much reflective attention to the present. Mindfulness practices are often aimed at 
improving a capacity to maintain attention to the present moment (Bishop et al., 2004), and to diminish habits of mind-wandering. By improving attention to the present moment in waking life, mindfulness may similarly increase the likelihood and frequency of lucid dream experiences. For example, one study (Stumbrys et al., 2015) showed that participants who reported having prior meditation experience also reported higher lucid dream frequency, with approximately 4.28 lucid dreams per month (vs. 2.55 in non-meditating study participants). In addition, participants who had prior meditation experience showed a significant correlation between mindfulness and lucid dreaming frequency. Thus, cultivating certain forms of focused attention in waking life may similarly elevate lucidity in dreaming.

Further, lucidity is not a discrete phenomenon, and levels of lucidity seem to vary across a continuum. Dreams can be considered semi-lucid when showing some levels of insight into the dream-state (Mallett et al.,

\begin{tabular}{|c|c|}
\hline I was aware that I was dreaming. & Why did you rate your awareness the way you did? \\
\hline Not at all & I believed it was reality whan I was dreaming and had no doubt that it wasn't. \\
\hline Just a little & $\begin{array}{l}\text { I had no idea I was asleep. } \\
\text { I had no idea I was dreaming, I only knew the situation was odd. } \\
\text { I was only partially aware that it was a dream, and only towards the end of the dream. }\end{array}$ \\
\hline Moderately & $\begin{array}{l}\text { The dream at moments felt real. } \\
\text { Because the things occurring in my dream were too bizarre to be real life. } \\
\text { I was aware that what was happening in the dream made no sense... }\end{array}$ \\
\hline Pretty much & $\begin{array}{l}\text {.. I was able to partially control it. } \\
\text { Because I realized I was dreaming. } \\
\text { Because I was aware that I was dreaming, but ... it took me a while to realize I was asleep. } \\
\text { I was mostly aware that I was dreaming but at times things felt more real. } \\
\text {... I was aware that I was dreaning but could not completely grasp the idea of what I was doing ... }\end{array}$ \\
\hline Very much & I was fully aware I was dreaming. \\
\hline
\end{tabular}

Table 1. Semi-lucidity interrogation. Representative defenses of why a participant picked each DLQ-1 response (i.e., lucidity level). (from Mallet et al., 2021, with permission).

2021). For instance, in Table 1, dreamers can demonstrate moderate levels of lucidity if they begin to notice, e.g., 'the things occurring in my dream were too bizarre to be real life' or become 'aware that what was happening in the dream made no sense.' In such cases, there is an element of sustained attention which seems to enable the dreamer to notice how their experience moment-to-moment differs from normal waking life, and which precedes the full insight that they are experiencing a dream. This is in contrast to completely nonlucid dreams, in which the dreamer fully accepts without question the dream, for example, one participant states, 'I believed it was reality when I was dreaming and had no doubt.' There is a lack of critical awareness or reflection on the dream experience. As in Bishop et al. (2004), lucidity as a form of mindfulness requires sustained attention, without which the moment-to-moment experience, however bizarre or unusual, is not questioned and the dreamer is not even capable of questioning it. 
In addition to variations in degree of awareness within the dream, lucid dreamers have variation in degree of control over the dream environment, which seems to rely on attentional control. In one recent study (Mallett, 2020), participants were invited to the laboratory and asked to study an experimental room that contained a variety of objects and were then asked to attempt to recreate this scene while in a lucid dream. Several lucid dreamers were able to call to mind and accurately remember elements of the experimental scene while in the dream, but they experienced varying degrees of success when attempting to transmute remembered objects into forms in the dream-world. Various techniques of attentional focus and intention could be applied to attempt to create dream objects. One participant closed their eyes in the dream, thought of an object, and then opened their eyes, and the object would appear. This lends further support to the idea that stability of the dream environment is dependent on what the dreamer is attending to, and therefore, whatever the dreamer is not attending to becomes labile. In this example, the dreamer makes use of this quality of attention-based perception in dreams and decides to "reset" the dream environment in order to make manifest an imagined object. In another example, a participant reported: "I saw the rattlesnake, it was mostly orange and black. The first time I saw the tail it had three black bands but when I looked again there were five, then seven and finally thirteen.” Thus, again, objects that are not being attended to appear to be unstable and often mutate in transitions between attention.

Thus levels of insight, attention and control vary in lucid dreams; the control of the actual dream environment is somewhat unique compared to waking attention and is sometimes termed 'supernatural control', being able to transform and mutate aspects of the dream-world at will, a power we cannot exercise in the real waking (external) world. While degrees of control over the self, one's thoughts, and where one directs attention seem relatively common in lucid dreams, this 'supernatural control' likely requires more skillful levels of sustained attention, focusing one's intention to project imagined content into the dream-world. It seems that practices of waking visualization may to some extent enable 'supernatural control' in dreaming, perhaps most commonly observed when applying lucid dreaming as a treatment for nightmares.

Lucid dream therapy is in fact one of the American Academy of Sleep Medicine's recommended treatments for nightmares, in which the aim is to re-write bad dreams from within the dream state as they are occurring, In contrast to IRT, discussed above, where memory for dreams is re-written during visualization and rehearsal practices in waking state. The goal of lucid dream therapy is to become lucid within a recurring nightmare, using the recurring features of the nightmare as signs to trigger insight (similar to examples above in which bizarre or unusual dream features can be 'noticed' in nonlucid dreams if/when the dreamer is paying attention, and this increases levels of lucidity). Once lucid, the dreamer first has the benefit of realizing that the ongoing nightmare is not 'real', it's just a dream. This initial shift in perspective allows the dreamer to feel less overwhelmed, decreasing negative emotion, and providing the time and space for the dreamer to re-orient towards the dream with an open or curious or empowered attitude, and use the increased cognitive capacity inherent in lucidity to reflect on actions rather than impulsively reacting to the dream. In general, the practice of using lucid dreams to modify nightmares seems to enable dreamers to engage in constructive and adaptive patterns of attention to and meditation of the dream world, in a manner more consistent with waking skillful attention. Below is an example in which the dreamer realizes they are dreaming through the act of noticing, attending to, a recurring dream scenario, and then decides to shift the dream to get out of a stressful situation.

I was learning to drive with my previous instructor, and there was also another instructor in the car. I remember feeling frustrated because my feet could not move to press the 
pedals and the car started to slow down. At this point I realized I was dreaming because this is a recurring dream I have had before and recognized it. After realizing it was a dream I stopped the car and got out. The dream ended with my getting out of the car. (Mallett et al, 2021)

In general, practices of lucid dreaming engage varying levels of attention, first through simply noticing one is dreaming, and further through directing attention, controlling behavior, and finally, leveraging the malleability of the dream world to one's advantage.

\section{Summary and conclusions}

In Section 1 we discussed the many ways in which attention to dreams and attention in dreams functions as a means of access to the dreamworld. Attention to dreams while awake is a strong enabling factor for remembering dreams. The ability to remember dreams may also depend on individual traits, such as a patterns of brain activation of the attentional circuits during both sleep and wakefulness, or mindfulness, either acquired through long-term meditation practice, or as a general psychological disposition. Practices such as recording, rehearsing and sharing dreams play an important role in shaping future dreams. In the following section we will discuss how attention works while dreaming. Attention in dreams while asleep has qualities of both perception and of imagination. The way the dreamworld appears to us depends directly on what is attended to. We argue that dream life does not have to correspond to the criteria for plausibility or reality, because we conceptualize dreaming as a performative, imaginative, creative activity, and not a mere rehearsal of memories or of waking life. The dreamer's attention is also dependent on stimuli from the outside world: the dream body reacts to the experiences of the sleeping body, and the dreaming mind incorporates outside stimuli into the dream narrative, albeit often in a distorted, transformed manner.

In Section 2 we have discussed how attention works as a mediator of the dream experience through changes in agency and awareness in both lucid and non-lucid dreams. Co-creative theory of dreaming, for example, places the dreamer as an active agent in the dream, able to reason within the dream scenario and to make choices. Emotional valence of dreams, on the other hand, may demand and direct attentional resources of the dreamer, by either narrowing (in case of nightmares) or expanding (in case of positive, euphoric dreams) the attentional capacity. Dream incubation practices in waking life allow the dreamer to take charge of the mental imagery of the dream by either focusing on desirable dream aspects or by actively rescripting dream narrative. Lastly, awareness and agency in lucid dreams opens up possibilities for controlling or modulating the dream, including an ability to rescript nightmare scenarios as they unfold.

William James famously wrote of the constitutive power of attention and agency for experience: “... each of us literally chooses, by his way of attending to things, what sort of universe he shall appear to himself to inhabit" (James, 1902; p.424). This poetic intuition appears to be even more literally applicable to dreams. If the enactive view of phenomenology of dreaming is right, then a dream is dynamically composed by the dreamer by bringing forth imagery, environments, characters and objects as the dream unfolds. Even though most dreams happen (or at least appear to happen) without any obvious volition or intention, and are not entirely controllable by the dreamer, it is possible to change, enrich or dedramatize the dream by engaging in different attentional practices both in wake and during the dream. Attention to dreams during wake, in form of 
paying attention to memory of dreams, by recording, rehearsing and sharing dreams functions as a means of access to and mediates potential qualities of future dreams. Active engagement with dream imagery, whereas the dreamer learns to own their experience, to recognize its different qualities and to modify aspects of it in order to re-encode and re-appropriate the dream, has a power to change how future dreams will unfold. Finally, learning to be aware of the dream during the dream, and to deploy and practice attentional skills in order to become the author of the dream narrative, provides the dreamer with cognitive tools for experiencing the dream as an expansive and positive mental space. 


\section{References:}

Arnulf, I. (2011). The 'scanning hypothesis' of rapid eye movements during REM sleep: a review of the evidence. Arch Ital Biol, 149 (4), 367-382. doi:10.4449/aib.v149i4.1246

Aserinsky, E. (1965). Periodic respiratory pattern occurring in conjunction with eye movements during sleep. Science, 150(3697), 763-766. Retrieved from http://www.ncbi.nlm.nih.gov/pubmed/5844080

Aspy, D. J. (2016). Is dream recall underestimated by retrospective measures and enhanced by keeping a logbook? An empirical investigation. Consciousness and Cognition, 42, 181-203. doi:10.1016/j.concog.2016.03.015

Aurora, R. N., Zak, R. S., Auerbach, S. H., Casey, K. R., Chowdhuri, S., Karippot, A., .. A American Academy of Sleep, M. (2010). Best practice guide for the treatment of nightmare disorder in adults.J Clin Sleep Med, 6(4), 389-401. Retrieved from https://www.ncbi.nlm.nih.gov/pubmed/20726290

Baird, B., Riedner, B. A., Boly, M., Davidson, R. J., \& Tononi, G. (2019). Increased lucid dream frequency in long-term meditators but not following MBSR training. Psychol Conscious (Wash D C), 6(1), 40-54. doi: $10.1037 /$ cns0000176

Beaulieu-Prevost, D., \& Zadra, A. (2004). How dream recall frequency shapes people's beliefs about the content of their dreams. North American Journal of Psychology, 7(2), 253-264.

Beaulieu-Prevost, D., \& Zadra, A. (2007). Absorption, psychological boundaries and attitude towards dreams as correlates of dream recall: two decades of research seen through a meta-analysis. Journal of Sleep Research, 16(1), 51-59. doi:10.1111/j.1365-2869.2007.00572.x

Bishop, S. R., Lau, M., Shapiro, S., Carlson, L., Anderson, N. D., Carmody, J., \& Segal, Z. V. (2004). Mindfulness: A proposed operational definition. Clinical psychology: Science and practice, 11(3), 230241.

Blagrove, M., Hale, S., Lockheart, J., Carr, M., Jones, A., \& Valli, K. (2019). Testing the Empathy Theory of Dreaming: The Relationships Between Dream Sharing and Trait and State Empathy. Front Psychol, 10, 1351. doi:10.3389/fpsyg.2019.01351

Carr, M. \& Nielsen, T. (2015). Daydreams and nap dreams: Content comparisons. Consciousness and Cognition, 36, 196-205

Carr, M., \& Nielsen, T. (2017). A novel Differential Susceptibility framework for the study of nightmares: Evidence for trait sensory processing sensitivity. Clin Psychol Rev, 58, 86-96. doi:10.1016/j.cpr.2017.10.002

Carr, M., Summers, R., Bradshaw, C., Newton, C., Ellis, L., Johnson, E. \& Blagrove, M. (2020). Frontal brain activity and subjective arousal during emotional picture viewing in nightmare sufferers. Frontiers in Neuroscience, 14, 1032

Cartwright, R. (2011). The Twenty-Four Hour Mind: the Role and Sleep and Dreaming in Our Emotional Lives. Oxford: Oxford University Press.

Cicogna, P. C., \& Bosinelli, M. (2001). Consciousness during dreams. Consciousness and Cognition, 10(1), 2641. doi:10.1006/ccog.2000.0471

Conduit, R., Crewther, S. G., \& Coleman, G. (2000). Shedding old assumptions and consolidating what we know: Toward an attention-based model of dreaming. Behavioral and Brain Sciences, 23(6), 924-928.

Damasio, A. R., Grabowski, T. J., Bechara, A., Damasio, H., Ponto, L. L., Parvizi, J., \& Hichwa, R. D. (2000). Subcortical and cortical brain activity during the feeling of self-generated emotions. Nature Neuroscience, 3(10), 1049-1056. doi:10.1038/79871

Dennett, D. C. (1976). Are dreams experiences? The Philosophical Review, 85(2), 151-171. 
Dresler, M., Wehrle, R., Spoormaker, V. I., Steiger, A., Holsboer, F., Czisch, M., \& Hobson, A. (2014). Neural correlates of insight in dreaming and psychosis. Sleep Med Rev, In press. doi:DOI:

10.1016/j.smrv.2014.06.004

Edwards, C. L., Ruby, P. M., Malinowski, J. E., Bennett, P. D., \& Blagrove, M. T. (2013). Dreaming and insight. Front Psychol, 4, 979. doi:10.3389/fpsyg.2013.00979

Eichenlaub, J. B., Bertrand, O., Morlet, D., \& Ruby, P. (2014). Brain reactivity differentiates subjects with high and low dream recall frequencies during both sleep and wakefulness. Cereb Cortex, 24(5), 1206-1215. doi:10.1093/cercor/bhs388

Eichenlaub, J. B., Nicolas, A., Daltrozzo, J., Redoute, J., Costes, N., \& Ruby, P. (2014). Resting brain activity varies with dream recall frequency between subjects. Neuropsychopharmacology, 39(7), 1594-1602. doi:10.1038/npp.2014.6

Fazekas, P., Nemeth, G., \& Overgaard, M. (2019). White dreams are made of colours: What studying contentless dreams can teach about the neural basis of dreaming and conscious experiences. Sleep Med Rev, 43, 84-31. doi:https://doi.org/10.1016/j.smrv.2018.10.005

Foulkes, D. (2014). Dreaming: a cognitive-psychological analysis: Routledge.

Freud, S. (1900/2010). The Interpretation of Dreams (J. Strachey, Trans.). New York: Basic Books.

Gallagher, S., Hutto, D. D., Slaby, J., \& Cole, J. (2013). The brain as part of an enactive system. Behav Brain Sci, 36(4), 421-422. doi:10.1017/S0140525X12002105

Hartmann, E. (1996). Outline for a theory on the nature and functions of dreaming Dreaming, 6(2).

Herman, J. H., Barker, D. R., \& Roffwarg, H. P. (1983). Similarity of eye movement characteristics in REM sleep and the awake state. Psychophysiology, 20(5), 537-543. doi:10.1111/j.1469-8986.1983.tb03008.x

Hill, C. E., \& Knox, S. (2010). The use of dreams in modern psychotherapy. International Review of Neurobiology, 92, 291-317. doi:10.1016/S0074-7742(10)92013-8

Hill, C. E., Knox, S., Crook-Lyon, R. E., Hess, S. A., Miles, J., Spangler, P. T., \& Pudasaini, S. (2014). Dreaming of you: Client and therapist dreams about each other during psychodynamic psychotherapy. Psychother Res. doi:10.1080/10503307.2013.867461

Hobson, J. A., \& McCarley, R. W. (1977). The brain as a dream state generator: an activation-synthesis hypothesis of the dream process. American Journal of Psychiatry, 134(12), 1335-1348. Retrieved from http://www.ncbi.nlm.nih.gov/pubmed/21570

Hobson, J. A., \& Voss, U. (2011). A mind to go out of: reflections on primary and secondary consciousness. Consciousness and Cognition, 20(4), 993-997. doi:10.1016/j.concog.2010.09.018

Hoeksma, J. B., Oosterlaan, J., \& Schipper, E. M. (2004). Emotion regulation and the dynamics of feelings: a conceptual and methodological framework. Child Dev, 75(2), 354-360. doi:10.1111/j.14678624.2004.00677.x

Holecek, A. (2016). Dream Yoga: Illuminating Your Life Through Lucid Dreaming and Tibetan Yogas od Sleep. Louisville: Sounds True.

Holzinger, B., Klösch, G., \& Saletu, B. (2015). Studies with lucid dreaming as add-on therapy to Gestalt therapy. Acta Neurologica Scandinavica, 131(6), 355-363.

Horton, C. L., \& Malinowski, J. E. (2015). Autobiographical memory and hyperassociativity in the dreaming brain: implications for memory consolidation in sleep. Front Psychol, 6, 874.

doi:10.3389/fpsyg.2015.00874

Hutton, S. B. (2008). Cognitive control of saccadic eye movements. Brain Cogn, 68(3), 327-340.

doi:10.1016/j.bandc.2008.08.021

James, W. (1902). The Principles of Psychology. Volume 1. New York: H. Holt and Company. 
Kahan, T. L., \& LaBerge, S. P. (2011). Dreaming and waking: similarities and differences revisited. Consciousness and Cognition, 20(3), 494-514. doi:10.1016/j.concog.2010.09.002

Kahan, T. L., \& Sullivan, K. T. (2012). Assessing metacognitive skills in waking and sleep: a psychometric analysis of the Metacognitive, Affective, Cognitive Experience (MACE) questionnaire. Consciousness and Cognition, 21(1), 340-352. doi:10.1016/j.concog.2011.11.005

Kozmová, M., \& Wolman, R. N. (2006). Self-awareness in dreaming. Dreaming, 16(3), 196-214.

Krakow, B., \& Zadra, A. (2006). Clinical management of chronic nightmares: imagery rehearsal therapy. Behav SleepMed, 4(1), 45-70. doi:10.1207/s15402010bsm0401_4

La Berge, S. P. (1980). Lucid dreaming as a learnable skill: A case study. Percept Mot Skills, 51(3), 1039-1042.

LaBerge, S., Baird, B., \& Zimbardo, P. G. (2018). Smooth tracking of visual targets distinguishes lucid REM sleep dreaming and waking perception from imagination. Nat Commun, 9(1), 3298. doi:10.1038/s41467-018-05547-0

Malinowski, J. E., \& Horton, C. L. (2014). Memory sources of dreams: the incorporation of autobiographical rather than episodic experiences. Journal of Sleep Research. doi:10.1111/jsr.12134

Mallett, R. (2020). Partial memory reinstatement while (lucid) dreaming to change the dream environment. Consciousness and Cognition, 83, 102974. doi:10.1016/j.concog.2020.102974

Mallett, R., Carr, M., Freegard, M., Konkoly, K., Bradshaw, C., \& Schredl, M. (2021). Exploring the range of dream lucidity. Phil Mind Sci, 2, 1-23. doi: https://doi.org/10.33735/phimisci.2021.63

Mamelak, A. N., \& Hobson, J. A. (1989). Dream Bizarreness as the Cognitive Correlate of Altered Neuronal Behavior in REM Sleep.J Cogn Neurosci, 1(3), 201-222. doi:10.1162/jocn.1989.1.3.201

Menary, R. (2010). Introduction to the special issue on $4 \mathrm{E}$ cognition. Phenomenology and the Cognitive Sciences, 9(4), 459-463.

Merleau-Ponty, M. (1945). Phénoménologie de la Perception. Saint-Amand: Gallimard.

Nielsen, T. (1993). Changes in the kinesthetic content of dreams following somatosensory stimulation of leg muscles during REM sleep. Dreaming, 3(2), 99-113.

Nielsen, T. (2000). A review of mentation in REM and NREM sleep: "covert" REM sleep as a possible reconciliation of two opposing models. Behav Brain Sci, 23(6), 851-866; discussion 904-1121. Retrieved from http://www.ncbi.nlm.nih.gov/pubmed/11515145

Nielsen, T. (2017). Microdream neurophenomenology. Neuroscience of Consciousness, 3(1), 1-17. doi: https://doi.org/10.1093/nc/nix001

Nielsen, T., \& Levin, R. (2007). Nightmares: a new neurocognitive model. Sleep Med Rev, 11(4), 295-310. doi:10.1016/j.smrv.2007.03.004

Nielsen, T., \& Stenstrom, P. (2005). What are the memory sources of dreaming? Nature, 437(7063), 12861289. doi: $10.1038 /$ nature 04288

Nielsen, T. A., \& Carr, M. (2017). Nightmares and nightmare function. In M. H. Kryger, T. Roth, \& W. Dement (Eds.), Principles and Practices of Sleep Medicine, 6e (pp. 546-554): Elsevier.

Noe, A. (2006). Action in Perception. Cambridge, MA: The MIT Press.

Norbu, N. (2002). Dream Yoga and the Practice of Natural Light, Revised: Snow Lion Publications.

Nummenmaa, L., \& Calder, A. J. (2009). Neural mechanisms of social attention. Trends in Cognitive Sciences, 13(3), 135-143. doi:10.1016/j.tics.2008.12.006

Pesant, N., \& Zadra, A. (2004). Working with dreams in therapy: what do we know and what should we do? Clin Psychol Rev, 24(5), 489-512. doi:10.1016/j.cpr.2004.05.002

Rasmussen, A. S., \& Berntsen, D. (2011). The unpredictable past: spontaneous autobiographical memories outnumber autobiographical memories retrieved strategically. Consciousness and Cognition, 20(4), 1842-1846. doi:10.1016/j.concog.2011.07.010 
Rechtschaffen, A. (1997). The single-mindedness and isolation of dreams. The Mythomanias: The Nature of Deception and Self-Deception, 203.

Rittenhouse, C., Stickgold, R., \& Hobson, A. (1994). Constraint on the Transformation of Characters, Objects, and Settings in Dream Reports. Consciousness and Cognition, 3(1), 100-113.

Roffwarg, H. P., Muzio, J. N., \& Dement, W. C. (1966). Ontogenetic development of the human sleep-dream cycle. Science, 152(3722), 604-619. doi:10.1126/science.152.3722.604

Saredi, R., Baylor, G. W., Meier, B., \& Strauch, I. (1997). Current concerns and REM-dreams: a laboratory study of dream incubation. Dreaming, 7, 195-208.

Sauvageau, A., Nielsen, T., \& Montplaisir, J. (1998). Effects of somatosensory stimulation on dream content in gymnasts and control participants: evidence of vestibulomotor adaptation in REM sleep. Dreaming, 8, $125-134$.

Schredl, M. (2002). Questionnaires and diaries as research instruments in dream research: Methodological issues. Dreaming, 12(1), 17.

Schredl, M., Atanasova, D., Hormann, K., Maurer, J. T., Hummel, T., \& Stuck, B. A. (2009). Information processing during sleep: the effect of olfactory stimuli on dream content and dream emotions. Journal of Sleep Research, 18(3), 285-290. doi:10.1111/j.1365-2869.2009.00737.x

Singer, J. (1966). Daydreaming: an introduction to the experimental study of inner experience. New York: Random House.

Smallwood, J., \& Schooler, J. W. (2006). The restless mind. Psychol Bull, 132(6), 946-958. doi:10.1037/00332909.132.6.946

Solomonova, E. \& Sha, X.W. (2016). Exploring the depth of dream experience: the enactive framework and methods for neurophenomenological research. Constructivist Foundation, 11(2), 407-416

Solomonova, E. (2017). Embodied mind in sleep and dreaming: a theoretical framework and an empirical stidy of sleep, dreams and memory in meditators and controls. (PhD). Université de Montréal, Montreal.

Solomonova, E., \& Carr, M. (2019). Incorporation of external stimuli into dream content. In K. Valli \& R. Hoss (Eds.), Dreams: understanding biology, psychology, and culture (Vol. 1). Santa Barbara, CA, USA: ABC-CLIO.

Spadafora, A., \& Hunt, H. T. (1990). The multiplicity of dreams: cognitive-affective correlates of lucid, archetypal, and nightmare dreaming. Percept Mot Skills, 71(2), 627-644. doi:10.2466/pms.1990.71.2.627

Sparrow, G. S. (2014). Analyzing chronic dream ego responses in co-creative dream analysis. International Journal of Dream Research, 7(1), 33-38.

Speth, C., \& Speth, J. (2018). A New Measure of Hallucinatory States and a Discussion of REM Sleep Dreaming as a Virtual Laboratory for the Rehearsal of Embodied Cognition. Cogn Sci, 42(1), 311-333. doi: $10.1111 / \operatorname{cogs} .12491$

Stewart, J., Gapenne, O., \& Di Paolo, E. (Eds.). (2010). Enaction: Toward a New Paradigm for Cognitive Science. Boston: MIT Press.

Stickgold, R. (2005). Why we dream. In M. H. Kryger, T. Roth, \& W. Dement (Eds.), Principles and practices of sleep medicine (pp. 579-587). Philadelphia: Elsevier.

Stickgold, R., \& Walker, M. P. (2013). Sleep-dependent memory triage: evolving generalization through selective processing. Nature Neuroscience, 16(2), 139-145. doi:10.1038/nn.3303

Stumbrys, T., Erlacher, D., \& Malinowski, P. (2015). Meta-awareness during day and night: the relationship between mindfulness and lucid dreaming. Imagination, Cognition and Personality, 34(4), 415-433.

Thompson, E. (2014). Waking, Dreaming, Being: Self and Consciousness in Neuroscience, Meditation, and Philosophy: Columbia University Press. 
Vallat, R., Eichenlaub, J. B., Nicolas, A., \& Ruby, P. (2018). Dream Recall Frequency Is Associated With Medial Prefrontal Cortex White-Matter Density. Front Psychol, 9, 1856. doi:10.3389/fpsyg.2018.01856

Varela, F. J., Thompson, E., \& Rosch, E. (1992). The Embodied Mind: Cognitive Science and Human Experience. Cambridge: MIT Press.

Wallace, B. A., \& Hodel, B. (2012). Dreaming Yourself Awake Lucid Dreaming and Tibetan Dream Yoga for Insight and Transformation: Shambhala Publications.

Wamsley, E. J., \& Stickgold, R. (2010). Dreaming and offline memory processing. Current Biology, 20(23), R1010-1013. doi:10.1016/j.cub.2010.10.045

Wegner, D. M., Wenzlaff, R. M., \& Kozak, M. (2004). Dream Rebound The Return of Suppressed Thoughts in Dreams. Psychological Science, 15(4), 232-236.

Windt, J. M. (2015a). Dreaming: A conceptual framework for philosophy of mind and empirical research. In: Cambridge: MIT Press.

Windt, J. M. (2015b). Just in Time-Dreamless Sleep Experience as Pure Subjective Temporality. In J. Windt \& T. Metzinger (Eds.), Open MIND. Frankfurt am Main: MIND Group.

Windt, J. M., Nielsen, T., \& Thompson, E. (2016). Does Consciousness Disappear in Dreamless Sleep? Trends in Cognitive Sciences, 20(12), 871-882. doi:10.1016/j.tics.2016.09.006

Wu, W. (2016). Experts and deviants: The story of agentive control. Philosophy and Phenomenological Research, 93(1), 101-126. 